\title{
La seguridad del paciente en atención primaria en salud ¿Una actividad que podría quedar en el olvido?*
}

\author{
The Patient Safety in the Primary Health Care. An Activity that Could be Overlooked? \\ A segurança do paciente em atenção primária à saúde. Uma atividade que poderia ficar no esquecimento?
}

Olga Lucia Moya Sáenz ${ }^{\text {a }}$

DOI: https://doi.org/10.11144/Javeriana.rgps17-34.spap

Universidad del Rosario, Colombia

ORCID: http://orcid.org/0000-0003-0402-861X

Fecha de recepción: 29 Marzo 2017

Fecha de aceptación: 16 Octubre 2017

\section{Resumen:}

La identificación de los riesgos en seguridad del paciente durante la atención primaria en salud habitualmente se realiza de la misma manera que se hace en la gestión clínica hospitalaria, sin tener en cuenta que los riesgos son diferentes. Del mismo modo, en Colombia no existe una política pública ni una directriz normativa sectorial que obligue a los actores del sistema a utilizar instrumento estandarizados, sistematizados para registrar, reportar y analizar los eventos adversos en atención primaria en salud. El gran reto del Sistema General de Seguridad Social en Salud está en prever la política y las herramientas adecuadas para asumir las grandes transformaciones que implica la implementación de la Política de Atención Integral en Salud, con una mirada hacia la atención primaria en salud, con un enfoque hacia la promoción de la salud y la prevención de la enfermedad, entornos que favorezcan la seguridad del paciente durante la prestación de los servicios en salud.

Palabras clave: atención primaria en salud, gestión de riesgo, seguridad del paciente, niveles de atención en salud, servicios hospitalarios.

\begin{abstract}
:
Identification of the safety risks to the patient during the primary health care is usually carried out in the same way as the hospital clinical management, without taking into account that different risks are involved in either case. In the same vein, in Colombia there is not any public policy or regulatory guidelines specific to this sector, intended to demand the involved actors the use of standardized systematic instruments to record, report and analyze the adverse events in the primary health care. The big challenge to the Social Security General System for Health is to provide both a policy and the appropriate tools to undertake great transformations. It implies the implementation of a Comprehensive Health Care Policy, paying special attention to the primary health care, focusing on both the health promotion and disease prevention, and ensuring an environment to favor the patient safety during the health care services.
\end{abstract}

Keywords: primary health care, risk management, patient safety, health care levels, hospital services.

Resumo:

A identificação de riscos em segurança do paciente durante a atenção primária à saúde habitualmente é realizada da mesma maneira em que se faz na gestão clínica hospitalar, sem levar em conta que os riscos são diferentes. De igual modo, na Colômbia nem existe uma política pública, nem uma diretriz normativa setorial que obrigue os atores do sistema a utilizar instrumentos padronizados, sistematizados para registrar, reportar e analisar os eventos adversos em atenção primaria à saúde. O grande desafio do Sistema Geral de Previdência Social em Saúde é prever a política e as ferramentas adequadas para assumir as grandes transformações que implica a implementação da Política de Atendimento Integral em Saúde, com olhar para a atenção primária à saúde, com abordagem para a promoção da saúde e a prevenção da docência e ambientes que favoreçam a segurança do paciente durante a prestação dos serviços em saúde.

Palavras-chave: atenção primária à saúde, gestão de risco, segurança do paciente, níveis de atenção em saúde, serviços hospitalares. 


\section{Introducción}

La seguridad del paciente (SP) es una de las dimensiones fundamentales de la calidad asistencial, ya que por ser considerada un elemento transversal, afecta a todas las organizaciones del Sistema de Salud y a sus miembros [1]. Según la Organización Mundial de la Salud (OMS), la seguridad es un principio fundamental de la atención al paciente y un componente crítico de la gestión de la calidad. Mejorarla requiere una labor compleja que afecta a todo el sistema, en la que interviene una amplia gama de medidas relativas a la mejora del funcionamiento, la seguridad del entorno y la gestión del riesgo [2].

María es una mujer de 86 años, con hipertensión, diabetes mellitus, obesa y con una gonartrosis severa bilateral, a quien recientemente le han diagnosticado una fibrilación auricular tras una descompensación cardíaca que requirió ingreso hospitalario. Acude a revisión a la consulta de enfermería, donde plantea una duda sobre la medicación para el dolor de rodillas. La enfermera pasa a la consulta médica y pregunta si María podría tomar más dosis de su antiinflamatorio no esteroideo habitual tras el alta hospitalaria. La médica, sin consultar la historia, le indica que mejor pruebe con otro fármaco como metamizol, ya que no interfiere con su hipertensión, ni con el nuevo anticoagulante que le han prescrito para la fibrilación. Sin embargo, María es alérgica al metamizol y la médica no lo recordó en ese momento.

La paciente presenta un cuadro anafiláctico por el que es ingresada de nuevo en el hospital. Una vez dada de alta, vuelve a la consulta a la semana siguiente. La médica de familia conoce el desenlace de la prescripción realizada en ese momento. Se siente abrumada y le dice cuánto siente lo que le ha pasado, iba deprisa, estaba atendiendo a otro paciente y no consultó la historia clínica antes de hacerle la recomendación [3]. Este caso puede corresponder a uno de los tantos eventos adversos (EA) que se presentan frecuentemente en el entorno de la atención primaria en salud (APS) en cualquier lugar del mundo y que no siempre son visibilizados.

Se entenderá APS como la puerta de entrada del sistema de salud y el lugar donde se operativiza la continuidad de la atención para la mayor parte de la población, la mayoría de las veces. Este es el concepto de APS más común en Europa y en otros países industrializados, su énfasis está en el primer nivel de atención. [4]. En el caso de Colombia, la Ley 1438 de 2011 define atención primaria en salud como:

“[... la estrategia de coordinación intersectorial que permite la atención integral e integrada, desde la salud pública, la promoción de la salud, la prevención de la enfermedad, el diagnóstico, el tratamiento, la rehabilitación del paciente en todos los niveles de complejidad a fin de garantizar un mayor nivel de bienestar en los usuarios, sin perjuicio de las competencias legales de cada uno de los actores del Sistema de Salud. [5]"

Desde hace más de veinticinco años se reconoce a la APS como uno de los componentes fundamentales de un sistema de salud efectivo, la situación actual requiere un examen exhaustivo de esta — tanto en la teoría como en la práctica一, así como una mirada crítica acerca de cómo puede "renovarse" con el fin de que refleje mejor las necesidades contemporáneas en materia de salud y de desarrollo de la población. Por tal razón, y por mandato de los Estados miembros de la Organización Panamericana de la Salud (OPS) mediante una resolución de 2003, se define la posición de la OPS con respecto a la propuesta de renovación de la APS. Su objetivo es aportar ideas y recomendaciones que hagan posible esta renovación y ayuden a fortalecer y dar nuevo ímpetu a la APS, de forma que pueda liderar el desarrollo de los sistemas de salud más allá del próximo cuarto de siglo [6].

La APS renovada debe estar sustentada en cuatro pilares. El primero, orientado a garantizar la cobertura universal y así mejorar la equidad sanitaria, continuar con una reforma en la prestación de servicios de salud que ofrezca servicios integrados e integrales. El segundo debe colocar como centro de atención a las personas y sus necesidades, logrando con ello una mayor satisfacción en la atención y una mejor adherencia y eficacia en los tratamientos, evitando el uso de servicios de mayor nivel de complejidad. El tercero debe orientarse a fortalecer el liderazgo participativo, para garantizar una mayor confiabilidad en sus dirigentes y finalmente crear reformas a través de políticas públicas que favorezcan efectivamente la salud de las comunidades [7]. 
Desde este punto de vista, el usuario debe ser el centro de los modelos de atención en salud enfatizados en brindar servicios con calidad y seguridad. Sin embargo, se encuentran algunas deficiencias en el acceso a la atención desde la APS, las cuales se describen como atenciones peligrosas y se dan cuando la falta de planeación en la implementación de los servicios facilita la aparición de EA o incidentes de seguridad, al igual que la aparición y perpetuación de infecciones nosocomiales [7].

En Colombia, desde de la década de los noventa, específicamente cuando se implementa la Ley 100 de 1993, se produce una fragmentación de los servicios de salud. Con la Ley 1438 de 2011 se evidencia una oportunidad en términos organizacionales de los servicios, en el sentido de avanzar en la constitución de los sistemas integrados de salud [8].

A fin de dar alcance a estas renovadoras políticas y sus estrategias en nuestro país, se adoptan los lineamientos, las prioridades y las estrategias del Plan Decenal de Salud Pública 2012-2021 (PDSP), la Política de Atención Integral en Salud (PAIS) y el Modelo Integral de Atención en Salud (MIAS). El eje principal del PDSP, la PAIS y el MIAS es la consolidación del modelo de aseguramiento a través de la introducción de la APS como puerta de entrada a los otros niveles de atención, ya bajo el control de las entidades promotoras de salud (EPS). A partir de las APS, los niveles de atención ahora van a ser integrados en rutas y redes integrales de atención en salud (RIAS), siempre bajo la "responsabilidad" de la EPS [9].

\section{Hechos y datos}

Las cifras sobre el volumen de atenciones y el impacto de los riesgos en la atención asistencial hablan por sí solas. De acuerdo con los registros individuales de prestación de servicios de salud (RIPS) reportados por las EPS de los regímenes contributivo y subsidiado al Ministerio de Salud y Protección Social, del periodo 2009-2015, contenidos en el Sistema Integral de Información de la Protección Social (SISPRO) [10] del Ministerio de Salud y Protección Social, 49314300 colombianos demandaron la prestación de 1563229793 atenciones, mientras que el Estudio Iberoamericano de Efectos Adversos (IBEAS), el primer trabajo a gran escala en América Latina que evaluó los incidentes que causan daño durante la presentación de los servicios de salud en 58 hospitales de cinco países (Argentina, Colombia, Costa Rica, México y Perú), concluyó que la prevalencia estimada de efectos adversos fue de $10 \%$, más de $28 \%$ de los efectos adversos desencadenaron discapacidad y $6 \%$ la muerte de los pacientes [11] .

Estos datos evidencian el alto riesgo a que se exponen los pacientes cuando reciben servicios en el ámbito hospitalario. El riesgo y la seguridad del paciente (SP) no son conceptos distintos sino situaciones de un continuo de mayor o menor grado de riesgo para el paciente. El riesgo y la seguridad forman un equilibrio dinámico representado por la línea sinuosa que los separa: cuando uno aumenta, el otro disminuye [12].

Pero, entonces, ¿qué es la gestión del riesgo en la atención asistencial? La gestión de riesgo es el conjunto de actividades destinadas a identificar, evaluar y tratar el riesgo de que se produzca un EA, con el objetivo de evitar o minimizar sus consecuencias negativas tanto para el paciente como para los profesionales y la propia institución de salud [13].

Colombia cuenta con una política en seguridad del paciente expedida en 2008, que es transversal al Sistema Obligatorio de Garantía de Calidad (SOGCS). La política cuenta con lineamientos para la implementación y las herramientas para promover la estrategia de la seguridad del paciente. No obstante, su enfoque está más direccionado hacia la gestión clínica hospitalaria, ya que se considera que los EA resultantes de la práctica clínica cotidiana pueden, y generalmente suelen provocar más morbilidad, aumento de la estancia hospitalaria, mayor demanda de servicios de salud, incapacidad o muerte, así como también conflictos relacionados con sobrecostos entre los prestadores de servicios de salud [13].

Es evidente que la SP se ha centrado más en la práctica clínica en el ámbito hospitalario que en la APS, cuya naturaleza es predominantemente ambulatoria. Las normas legales vigentes que ordenan a las 
instituciones prestadoras de servicios de salud (IPS) de Colombia los contenidos mínimos para la habilitación y la acreditación de estas instituciones tampoco contemplan explícitamente acciones orientadas a garantizar la seguridad del paciente en APS [14]. Igualmente, el Ministerio de Salud y Protección Social de Colombia ha diseñado y divulgado veintitrés paquetes instruccionales dirigidos a los prestadores de servicios de salud [15] para que implementen, con carácter obligatorio, las mejores prácticas en SP en la atención en salud. Sin embargo, ninguno de estos paquetes está dirigido específicamente a las actividades asociadas a la APS, hecho que posiblemente se deba a que los esfuerzos de seguridad pueden ser más fáciles de implementar en el entorno hospitalario [16] y su extensión a actividades no hospitalarias depende de la voluntad, de la interpretación y de los recursos del prestador. De hecho, el paciente que recibe cuidados médicos en este entorno tiene la ventaja de que la mayoría de las veces los recibe por parte del mismo proveedor de servicios de salud, el cual tiene la posibilidad de realizar un seguimiento sistemático y continuo, lo que facilita que la atención en salud se realice entornos seguros y favorables.

Estos hechos no se deben convertir en una barrera que limite al paciente que va a recibir servicios de salud en APS para que ello se haga en condiciones de seguridad; al contrario, debe generar una gran expectativa al determinar qué tareas se deben desarrollar en la búsqueda de una prestación de servicios favorable para el paciente, la cual permita que el acceso a este servicio no cause ningún daño a su integridad.

En consecuencia, es claro que los riesgos que se presenten en APS y en la gestión clínica pueden conllevar los mismos desenlaces, incluso fatales. Desde esta perspectiva, los riesgos potenciales para la seguridad del paciente en un establecimiento de atención primaria son diferentes de los riesgos para la seguridad del paciente en un establecimiento de cuidados agudos. Las principales diferencias surgen de las estructuras organizativas de la APS en la prestación de los servicios de salud y en la mayor participación de los pacientes en su cuidado [17]. Dadas estas condiciones, el abordaje de este artículo se centra en analizar en qué medida el Sistema General de Seguridad Social en Salud promueve la gestión de los riesgos para la seguridad del paciente en APS.

Para lograr este propósito el artículo se divide en cuatro partes, en las cuales se intenta establecer, en primer lugar, la relación de la seguridad del paciente y la identificación de riesgos en el escenario de la APS; en segundo lugar, la importancia de conocer los eventos adversos e incidentes y su frecuencia; en tercer lugar, los elementos estructurales para hacer de la seguridad del paciente parte de la cultura organizacional de las instituciones y los beneficios que se logran al incorporar estos conceptos; y finalmente, en cuarto lugar, las características que deben tener los profesionales de la salud que trabajen en APS frente a la seguridad del paciente y la identificación de riesgos asistenciales.

\section{La seguridad del paciente y su relación con la APS}

La SP se considera una prioridad en la asistencia sanitaria, actividad cada vez más compleja que entraña riesgos potenciales y en la que no existe un sistema capaz de garantizar la ausencia de EA. Los EA ligados a la asistencia son un problema frecuente en la práctica clínica en cualquier nivel de cuidados [13].

Por esta razón, al no contar con un sistema capaz de identificar los riesgos, para posteriormente tratarlos, el impacto en el paciente puede ser muy alto. Se hace necesario que el objetivo primordial de la seguridad del paciente en APS sea mejorar la calidad de la atención, haciendo uso de las herramientas normativas y gerenciales dispuestas para tal fin.

Los datos disponibles de diversos estudios desarrollados en la Unión Europea muestran que entre el 8\% y el $12 \%$ de los pacientes hospitalizados presenta un EA asociado a la atención sanitaria. El impacto que los EA tienen sobre los pacientes, la carga económica que suponen para los servicios sanitarios y la evidencia disponible sobre las medidas para prevenirlos han alertado a los Estados miembros sobre la importancia de incluir la seguridad del paciente en la agenda de sus políticas sanitarias [18]. 
Por tal razón, es necesario tener en cuenta que la atención del paciente agudo se realiza en un ambiente hospitalario, generalmente en la misma organización, mientras que la APS puede brindarse por diferentes proveedores de servicios, lo que en ocasiones deriva en la pérdida de la continuidad de la información y del manejo de estos pacientes [17] y, de alguna manera, se expone al paciente a riesgos que deben ser identificados por las instituciones para su priorización y gestión.

Cabe resaltar que la APS es la entrada del paciente al sistema de salud, por ello es necesario tener a disposición de los pacientes un grupo interdisciplinario de profesionales que cubra sus necesidades de atención en salud. Los servicios que se brindan en APS incluyen promoción y prevención de la enfermedad, educación en hábitos saludables, diagnóstico, tratamiento, rehabilitación y cuidados paliativos. Al prestar esta variedad de servicios se dificulta ejercer control sobre estos por parte de los prestadores, ya que los pacientes son de diferentes rangos de edades, con gran variedad de condiciones de salud, alimentación y acceso a servicios de salud.

Debido a lo expuesto anteriormente, los programas de seguridad del paciente han enfatizado en los procesos asistenciales hospitalarios. De hecho, no existe una directriz normativa institucional que obligue a los prestadores a implementar programas de seguridad del paciente en APS ni un instrumento validado para el registro sistematizado de los EA que ocurren en APS [11]. Esta es una razón de peso para considerar que los eventos en salud que pueden poner en riesgo la vida de los pacientes no ocurren solamente en el proceso de atención asistencial en el contexto hospitalario.

En 2008, la Organización Mundial de la Salud (OMS) hizo una revisión de las publicaciones sobre seguridad de los pacientes en APS y estimó que la tasa de incidentes de seguridad ocurridos variaba enormemente, entre $0.004 \mathrm{EA}$ y $240 \mathrm{EA}$ por cada 1000 consultas, con un porcentaje de errores prevenibles entre el $45 \%$ y el $65 \%$ de todos los eventos [19]. En Colombia no se conocen estadísticas asociadas o que muestren la magnitud de incidencia y prevalencia de EA asociados a la prestación de servicios de APS.

Datos de la OMS sobre métodos e instrumentos de medición en la investigación sobre seguridad del paciente se han enfocado, principalmente, en identificar errores y EA en los hospitales. Sin embargo, no se ha investigado en profundidad en los ámbitos extrahospitalarios, como la atención domiciliaria, las consultas en dispensarios o las consultas a médicos generales o farmacéuticos. No hay mediciones de la seguridad del paciente en esos ámbitos. En general, en aquellos ámbitos en que los datos son más escasos faltan métodos de investigación eficaces [20].

Los datos de la OMS no solo han identificado esta situación [11]. La investigación sobre seguridad se ha centrado en hospitales, aunque en los últimos años el interés en APS se ha incrementado, sobre todo en economías emergentes en las que el primer nivel de atención ocupa un lugar preponderante en la atención en salud. ¿Acaso un error en el diagnóstico de un paciente en APS no puede llevar a un EA?

Un reciente informe del Instituto de Medicina (Institute Of Medicine [IOM]) en Estados Unidos y su programa Mejorando el Diagnóstico en la Atención Sanitaria destaca las implicaciones de seguridad de los errores de diagnóstico, que son uno de los tipos más comunes de errores médicos en APS. El informe estima que estos errores afectan anualmente en Estados Unidos a uno de cada veinte pacientes adultos ambulatorios, y llevan a la mala práctica ambulatoria, errores de diagnóstico que son sujetos de investigación en seguridad del paciente [21].

Por esta razón, la seguridad del paciente y la gestión de riesgo en APS deben tener otro énfasis y hacer frente a los retos de medir las consecuencias de EA que pongan en riesgo la integridad y la vida de los pacientes. Un estudio de revisión sistemática de la literatura de artículos publicados en Estados Unidos, Canadá y Europa [22] plantea que los errores en diagnóstico y prescripción constituyen los EA en APS más frecuentes. Los errores de diagnóstico varían entre el $4 \%$ y el $45 \%$ de los casos, y para errores de prescripción varían entre el 1\% y el 90\%. Estos errores son mayores en algunos subgrupos como pacientes mayores y pacientes polimedicados. No solo se debe entender la seguridad del paciente y la gestión de riesgo como no causar daño al paciente dentro del proceso de atención médica, sino que además es necesario contemplar los EA relacionados con 
la gestión o los trámites administrativos, como un diagnóstico tardío por inoportunidad en consulta por especialista; una accesibilidad limitada al sistema por barreras geográficas, económicas, administrativas o culturales, entre otras; una restricción de costos a tecnologías no cubiertas por el plan de beneficios, hechos que ponen en riesgo la vida y la integridad del paciente y su familia. Dada la necesidad de conocer los eventos y la frecuencia con que se presentan los EA en APS, España llevo a cabo el Estudio de Seguridad de los Pacientes en Atención Primaria de Salud (APEAS) e identificó una tasa de prevalencia de 11.2 EA por cada 1000 visitas a la asistencia sanitaria. De los EA graves, $70 \%$ eran evitables y la mayor parte estaban ligados a la medicación y a los cuidados, $24.6 \%$ a la comunicación y $8.9 \%$ a la gestión. La práctica sanitaria en AP es segura, pero si se generalizan los resultados a los profesionales de AP, cada paciente potencialmente podría sufrir seis EA en un año [23].

\section{Desconocimiento de los eventos en salud que se presentan en APS}

La seguridad del paciente en APS es un campo en desarrollo, con una base de pruebas embrionarias, pero en evolución. En el informe de la Asociación Médica de Estados Unidos sobre la seguridad del paciente ambulatorio [24], en 2011, se concluyó que la introducción y la investigación en SP en el entorno de atención primaria han quedado a la zaga de la atención secundaria. De esta manera, es importante conocer qué métodos, herramientas e indicadores se están utilizando en APS para medir la seguridad el paciente. Existen experiencias como MERIS (Medical Error Reporting Information System), sistema que ha sido evaluado de manera integral y eficaz, el cual tuvo éxito en la detección de los principales factores que causan eventos adversos y divulga omisiones graves en el sistema de salud griego. MERIS se incorporó y ejecutó de manera eficiente a nivel nacional, adaptado a las necesidades y peculiaridades de cada hospital o clínica [25].

No obstante lo anterior, el Gobierno español, a través de su Ministerio de Sanidad, Servicios Sociales e Igualdad, en colaboración con las comunidades autónomas en atención primaria en salud, cuenta con sistemas de notificación y de aprendizaje de incidentes sin daño, relacionados con la medicación, con retroalimentación a sus profesionales a través de boletines, pero con gran dificultad en su operación, ya que existen reticencias de los profesionales a notificar por el temor a posibles consecuencias punitivas [23].

Por tanto, los sistemas de notificación de eventos adversos para APS no deben ser los mismos que se utilizan en la gestión clínica, debido a que los riesgos que se deben identificar nacen de la particularidad de los servicios que se prestan. Es posible mencionar el caso exitoso de Alemania que, teniendo una necesidad imperiosa para determinar las diferencias entre la atención primaria y la secundaria, hizo un estudio en donde aplicó un cuestionario a 1800 médicos y asistentes de cuidado de la salud, seleccionados al azar en APS. Por ello, se realizó un estudio con el objetivo de identificar fortalezas y debilidades del clima de seguridad en este entorno y, adicionalmente, para identificar las características individuales y de práctica que afectan la percepción del clima de seguridad de la asistencia sanitaria de los profesionales de la atención primaria [26]. Se concluyó que los resultados de los pacientes hospitalizados no se pueden ajustar ni transferir a la APS.

La preocupación por desarrollar una herramienta de captura que permitiera identificar los EA y los incidentes que se presentan durante la asistencia de servicios en salud llamó la atención de la Oficina Regional de la Organización Panamericana de la Salud (OPS/Washington). Ésta en colaboración con la OPS/México y la Comisión Nacional de Arbitraje Médico de este mismo país efectuaron un estudio [11] con el objetivo de analizar los sistemas de notificación que operan en América Latina.

Cada uno de esos sistemas responde a objetivos y necesidades nacionales. Las experiencias de los países de la región y de fuera de ella muestran una gran variedad de modalidades operacionales de estos sistemas, lo cual refleja la diversidad de alternativas existentes. El estudio señaló que aún no existen evidencias científicas concluyentes sobre las bondades de un sistema en relación con otro, aunque sí existe un amplio consenso sobre el carácter de aprendizaje no punitivo que debe caracterizar a estos sistemas [11]. 
En Colombia existe el aplicativo para registro y gestión de EA, el cual fue gestionado por la OMS, la Organización Panamericana de la Salud (OPS) y el Ministerio de Salud y Protección Social. Este aplicativo tiene como finalidad el registro, la clasificación, el análisis de los EA, la gestión de los casos y la respectiva implementación de acciones de mejora [27]. No obstante, y para sorpresa de los profesionales de salud que trabajan en los programas de SP, este aplicativo no funciona de manera masiva y permanente en las IPS, debido posiblemente a que la gran mayoría cuenta con su propio sistema de notificación y reporte de EA. Al no contar con un sistema de notificación de EA estandarizado, se limita la provisión de datos e información confiable al sistema de salud para que conozca el panorama de riesgo sobre la SP en el país y con ello una toma de decisiones informada.

En este sentido, no contar con un sistema de información unificado y propio para la notificación e identificación de riesgos en la seguridad del paciente en APS se convierte en la mayor limitante para implementar barreras de seguridad o controles que mitiguen los riesgos. Del mismo modo, dificulta reducir la frecuencia de los problemas relacionados con la SP, ya que se hace imprescindible conocer las causas de los EA y diseñar estrategias para prevenirlos o minimizar sus consecuencias, todo ello al amparo de una política pública sectorial. Por esta razón, es necesario que la seguridad en la APS sea un componente fundamental de la calidad asistencial, que fundamentalmente consiste en no dañar, siendo conscientes de que siempre cabe el riesgo mínimo, irreductible pero asumible, que implica la asistencia sanitaria [12].

\section{La seguridad del paciente y la gestión de riesgos en APS, un elemento esencial de la cultura organizacional}

En este contexto, es necesario apropiar los conceptos de SP para incorporarlos en las organizaciones que prestan servicios o gestionan el aseguramiento en salud y orientarlos a fortalecer los procesos del direccionamiento estratégico, pues permite que el enfoque de SP se encuentre explícito en sus plataformas estratégicas, como la misión, la visión, los objetivos y las políticas, entre otros elementos, y tal vez lo más importante, desde el mandato de la alta dirección. En esta medida, es importante incorporar conceptos de SP como parte de la cultura organizacional, lo que permite a las instituciones establecer los mecanismos para identificar, reportar, analizar, intervenir y realizar las acciones de mejora que deben tener un seguimiento y evaluación sistemática y periódica.

Así, construir una cultura de seguridad (CS) en las instituciones de salud es fundamental para conseguir que la SP forme parte de la metodología y de las prácticas de trabajo cotidianas y de mejora continua de la calidad en los servicios asistenciales, y para ello es necesario que cada institución de salud conozca cuál es la situación de partida, a fin de saber cuáles son los aspectos que conviene priorizar [28].

Para que la cultura en SP sea un elemento esencial de la cultura organizacional, es necesario ofrecer servicios con calidad, enfocados en evaluar de manera sistemática y objetiva cuáles son los riesgos en SP que interfieren en el logro de este objetivo; por tanto, la calidad y la seguridad de la atención en salud de los pacientes es un binomio que muestra resultados convergentes. Si no hay calidad, no puede haber seguridad, y, en sentido contrario, sin seguridad no puede haber calidad en la prestación de los servicios de salud. La calidad establece los elementos necesarios para disminuir los riesgos en la operación diaria de las unidades de atención médica $y$, por ende, de lo que el paciente recibe en el proceso [29].

De la misma manera, a continuación se presentan algunas experiencias, a través de las cuales se pretende conocer el punto de partida de algunas instituciones para identificar los riesgos en la seguridad del paciente en APS. En las 61 unidades de atención primaria evaluadas por Fundación Pública Adscrita a la Consejería de Salud de la Junta de Andalucía (ACSA) en España se evaluaron tres dimensiones de la prestación de servicios. La primera se relaciona con la utilización de un método sistemático y estructurado de identificación proactiva de riesgos y su priorización, la segunda hace referencia a los riesgos potenciales para la seguridad del paciente 
identificados por las unidades, y la tercera, a las soluciones aportadas para evitar su aparición. Los resultados demostraron que tan solo el $50 \%$ las unidades cumplen el estándar analizado. La explicación de este hallazgo puede basarse en el hecho de que la preocupación por la seguridad del paciente, a pesar de su larga existencia, es relativamente nueva en el ámbito asistencial [13]. En otras palabras, a pesar de los esfuerzos, continúa siendo prematura la identificación de los riesgos en APS con respecto a la seguridad del paciente. La mayoría de las experiencias ponen en contexto las grandes dificultades de implementar un instrumento estandarizado sistemático para registrar, reportar, analizar y dar soluciones a los eventos adversos en las unidades de APS.

Esta problemática no puede ser una limitante para alcanzar el logro de los objetivos. El Instituto Mexicano de Seguridad Social (IMSS) aplica actualmente un sistema de gestión de calidad y seguridad del paciente, mediante estándares homologados para la Certificación de Hospitales del Consejo de Salubridad General, los cuales han sido establecidos por el Sistema Nacional de Certificación de Establecimientos de Atención Médica. El comité local de investigación en salud mexicano diseñó una herramienta que incluía el registro de fallas en la estructura y fallas en el proceso, validando el proceso de atención médica, la competencia profesional y los factores inherentes al paciente y a la cultura de la organización [11].

Como resultado de esta validación se instauró el Sistema de Registro y Notificación de Incidentes y Eventos Adversos para Unidades de Medicina Familiar (VENCER-MF) [11]. El propósito de los sistemas de notificación de eventos adversos e incidentes en APS no solo se limita a generar datos o estimaciones de prevalencia, incidencia, clasificación de los eventos en salud, sino que deben trascender y convertirse en una herramienta de mejora continua en el interior de las organizaciones que permita fortalecer la cultura en seguridad del paciente en la dinámica organizacional, articulado con sus sistemas de gestión de la calidad.

Hoy en día España presenta un claro ejemplo en avances sobre SP en APS. De acuerdo con el Plan de Calidad para el Sistema Nacional de Salud (SNS) de España para el periodo 2005-2009, se buscaba "mejorar la SP de los pacientes atendidos en los centros sanitarios del SNS”. El Ministerio de Sanidad de España, en colaboración con las comunidades autónomas, elaboró la Estrategia de SP del SNS 2005-2011 y financió estudios para conocer su realidad.

Después de diez años del despliegue de esta estrategia nacional han hecho una serie de propuestas para seguir construyendo el futuro de la SP en APS, centradas en seis objetivos: 1) promover y desarrollar el conocimiento y la cultura en seguridad del paciente; 2) diseñar y establecer sistemas de información y notificación de incidentes relacionados con la seguridad del paciente; 3) implantar prácticas seguras en el Sistema Nacional de Salud; 4) promover la investigación en seguridad del paciente; 5) participación de los pacientes en la estrategia de seguridad del paciente; 6) reforzar la participación de España en todos los foros sobre seguridad del paciente de las principales organizaciones internacionales [23].

La implementación de estas estrategias requiere niveles de planificación que garanticen la SP, en función del ámbito de responsabilidad en que se toman las decisiones. Se pueden definir diferentes niveles y características dentro de la organización, en las cuales es fundamental involucrar a todos los actores que emergen dentro del proceso de atención, cuya finalidad será generar entornos más seguros, para lo cual se orientan dichas estrategias en macrogestión, relacionada con la toma de decisiones en el ámbito más alto del Ministerio de Sanidad y de las consejerías de salud, donde se definen las políticas sanitarias; mesogestión, consistente en aproximar las decisiones de las políticas sanitarias en el ámbito de las organizaciones e instituciones, asegurando un funcionamiento eficiente y efectivo de los recursos disponibles; y microgestión, asociada con la toma de decisiones en el ámbito de los centros de salud, con los profesionales que están involucrados directamente con la atención del paciente, relacionados con la "gestión clínica". Por tanto, se busca aplicar los conocimientos de forma más efectiva y segura para el paciente. El centro de la atención es el paciente, por lo que es importante su participación en la toma de decisiones, todo ello alineado con las políticas sanitarias de la organización en términos de equidad, eficiencia y accesibilidad [19].

Finalmente, el resultado de la implementación de estas estrategias deberá consolidar y alinear a las organizaciones con el direccionamiento estratégico, en un enfoque de gestión centrado en la identificación 
de riesgos hacia el paciente y el mejoramiento continuo de la calidad asistencial, para alcanzar una cultura organizacional centrada en la SP, habida cuenta que la madurez de una organización se puede apreciar en la forma en que enfrenta sus problemas.

\section{El rol de los profesionales de salud en la identificación de riesgos en los programas de seguridad del paciente en APS}

A lo largo del artículo se ha mostrado la importancia de la SP y la gestión de riesgos en APS, la necesidad de contar con un lineamiento normativo sectorial de obligatorio cumplimiento para los prestadores, un sistema de notificación de eventos en salud, la transformación de la cultura organizacional en seguridad del paciente y los elementos estructurales fundamentales para generar entornos favorables para los pacientes. Sin embargo, el factor humano es indispensable en este gran desafío. De hecho, existe un enfoque de competencias para los equipos de APS.

Una revisión de la literatura que buscaba indagar por las competencias para la APS, acudió a la visión holística de la OPS, la cual establece que las competencias para un adecuado desempeño de los integrantes de los equipos de APS implican que estos tengan conocimientos (saber) para entender y ejecutar una labor; habilidad para poner en práctica los conocimientos en una problemática o actividad específica (saber hacer); estar motivados y tener actitud (querer hacer), y, finalmente, disponer de los medios y recursos necesarios (poder hacer). Se encontró que diversos autores reconocen que los cambios acelerados en el entorno de las organizaciones, incluidas las del sector salud, han generado la necesidad de centrar la gestión del talento humano en las competencias requeridas para participar y enfrentar sociedades, organizaciones y problemáticas complejas [30].

Debido a que la SP es un proceso complejo, son los profesionales de la salud y los equipos de salud los que tienen un reto superior, el de incentivar el desarrollo de ciertas competencias y habilidades propias, en las cuales los pacientes encuentren los espacios más seguros para su proceso de atención. Por tal razón, la SP es resultado de la interacción y el equilibrio permanente, por un lado, de una serie de condiciones latentes que incluyen la cantidad y la calidad de los recursos, la llamada CS y las características del contexto institucional, y por otro lado, de los profesionales y los equipos que desarrollan su actividad en el entramado asistencial [12].

Otra de las experiencias que aportan elementos para identificar las competencias que deben poseer los profesionales de salud que trabajan en APS, se encontró en Escocia, en una investigación llevada a cabo por el Sistema Nacional de Salud (National Health System [NHS]), la cual consistió en aplicar una encuesta semiestructurada a profesionales del área de la salud vinculados APS. Las conclusiones de dicho estudio se enmarcaron en tres dimensiones: percepciones, experiencias y beneficios; mejoras en los sistemas de atención al paciente y la utilidad de las intervenciones. Sin embargo, se identificaron algunos desafíos relacionados con la comprensión de conceptos y la pertinencia en la aplicación de algunas intervenciones de mejora probadas con respecto a la implementación social y técnica, por ejemplo, el uso adecuado de los incentivos financieros y la disponibilidad de los datos y las demandas de carga de trabajo [31].

A pesar de que existen experiencias positivas del rol del profesional de SP en APS, todavía se encuentran deficiencias en la identificación y notificación de EA, debido al el temor a las represalias de parte de las organizaciones. Es claro que la importancia de la notificación de los EA e incidentes guarde la confidencialidad y la absoluta reserva, con la finalidad de brindar espacios de confianza, y que sean los propios profesionales de la salud quienes intervengan directamente en las acciones de mejora puntuales. Otro de los retos de las instituciones de salud con respecto a los profesionales es el adecuado manejo de los incentivos frente al reporte; estos deben estar direccionados estrictamente hacia la mejora continua de los procesos y no estar vinculados a intereses personales o financieros de las organizaciones. En este sentido, se hace necesario formar a los profesionales de la salud y a los equipos de salud en SP en APS, enmarcada en una continua y permanente 
educación sobre conceptos en SP, direccionados a identificar los riesgos propios del proceso. Esto permitirá una adecuada notificación de los EA o incidentes. Para la Consejería de Sanidad de la Comunidad de Madrid (CSCM), la formación de los profesionales en SP ha conformado desde sus inicios una línea estratégica dentro de su nueva estructura organizativa y funcional creada en 2010, con el propósito de brindar cobertura en salud en APS a la comunidad de Madrid. Esta línea estratégica es una prioridad, pero del mismo modo permite integrar los cursos y los contenidos de estos que son ofertados a todos los profesionales en forma de talleres, sesiones y jornadas. Así mismo, se imparte formación dirigida a los residentes de medicina familiar y comunitaria, al ser considerada esta formación por parte de la organización como actividad obligatoria dentro de su programa formativo [32].

\section{Discusión}

La APS es un elemento clave de los sistemas sanitarios. Su mayor proximidad a la población, la longitudinalidad de la atención que ofrece y la orientación comunitaria de sus servicios hacen de ella el escenario ideal para las intervenciones de promoción de la salud y de prevención de la enfermedad. En la actualidad la fuerte crisis económica, junto con el envejecimiento de la población y el consiguiente aumento de la prevalencia de las enfermedades crónicas, está llevando a diferentes países a considerar una reforma en sus sistemas sanitarios basados en APS, con el objetivo de hacerlos más eficientes, disminuir las desigualdades en salud [33] y, paralelamente, fortalecer su sostenibilidad financiera. Enfrentar estos grandes retos en los sistemas de salud se convierte en una necesidad imperiosa de proveer mejoras en la seguridad de los pacientes, ejemplo de ello se encuentra en el Sistema Nacional de Salud (SNS) español, con la creación del Observatorio Regional de Riesgos Sanitarios (ORRS) en el año 2006. El ORRS es un instrumento de apoyo a la gestión de riesgos sanitarios derivados de la práctica asistencial, mediante la información y el análisis de estos, proponiendo medidas encaminadas a evitarlos o, en su caso, a la corrección de las causas que los ocasionan, y entre sus objetivos se incluye la creación de una estructura organizativa para la gestión de riesgos sanitarios. Como resultado de esta línea de trabajo, en la comunidad de Madrid se constituyeron las unidades funcionales de gestión de riesgos sanitarios (UFGRS) y se han elaborado los distintos planes de riesgos, así como la estrategia de seguridad del paciente (SP) actual 2010-2012. Se describen las prácticas seguras elaboradas por la UFGRS en Atención Primaria (AP), desde su creación hasta la actualidad. Igualmente, se identifican las prácticas seguras simples (PSS) como aquellas que tienen un mejor equilibrio entre el impacto en la SP y la complejidad de su implantación [34]. Básicamente, lo que buscaba el estudio era comparar las prácticas seguras simples en el ámbito asistencial, con relación a las posibles prácticas seguras simples en el ámbito de AP. En consecuencia, se puede afirmar que la gestión de riesgos en SP en la práctica asistencial es diferente a la que se presenta en el ámbito de la APS, lo que permite determinar, según el estudio 'Identificación de eventos centinela en atención primaria', que el número total de eventos centinela (EC) identificados en AP es menor [16] que el de los incluidos en el ámbito hospitalario [27]. Esto resulta coherente con el mayor nivel de complejidad del hospital con respecto a AP, donde aparecen muchos EC en relación con los actos quirúrgicos. Igualmente, en su adaptación no se incluyeron los EC considerados actos criminales, debido a lo realmente excepcionales que resultan en el ámbito de la AP, donde el paciente tiene un tiempo de permanencia muy limitado. Los EC propuestos como específicos para la AP fueron sucesos de carácter concreto, con diferencias sutiles en las redacciones, y por tanto con la misma esencia, lo que permitió unificarlos de manera global fácilmente. La mayoría de los descritos estaba en relación con la medicación o las vacunas, algo que tiene que ver con que un porcentaje elevado de eventos adversos notificados en AP son errores de medicación. Sin embargo, aparece como EC un grupo de eventos en relación con el diagnóstico, categoría no incluida en el listado de EC de hospitales. Estos EC tienen que ver tanto con pruebas diagnósticas, retrasos en la petición o en la visualización de datos a tiempo, cuando son verdaderas alertas, así como con retrasos en el 
diagnóstico de enfermedades graves. Con respecto a los problemas en relación con las pruebas diagnósticas, el listado actualizado del National Quality Forum (NQF) de 2011 incluyó en la categoría de cuidados un nuevo EC: muerte del paciente o lesiones graves como resultado de la falta de seguimiento o comunicación de pruebas de laboratorio, patología o radiología. En cuanto a las demoras en el diagnóstico de las enfermedades graves en AP influyen diferentes factores: la variabilidad en la precisión diagnóstica de los médicos, la propia enfermedad, el paciente y el profesional. Así, algunas enfermedades son difíciles de diagnosticar y en otras ocasiones podría deberse a la falta de conocimiento por parte del profesional o al cierre prematuro de la historia clínica [35].

Es posible que en Colombia se requiera replantear las políticas públicas y las prioridades que tiene el Estado para lograr cobertura universal y equidad [36], a efectos de fortalecer la probabilidad del logro en cuanto al cumplimiento de los atributos de calidad direccionados por el Sistema General de Seguridad Social en Salud (SGSSS), como pertinencia, accesibilidad, continuidad, oportunidad y seguridad, que se acentúan por la franca fragmentación en el momento de la prestación de los servicios, lo cual contribuye a la materialización de EA, ya sea en el ámbito de la APS, o bien en el de la gestión hospitalaria.

Debe avanzarse en la constitución de los sistemas integrados de salud. Como respuesta a la tan mencionada fragmentación que dejaron las reformas de los años noventa, en Colombia, específicamente, la implementación de la Ley 100 de 1993. Al respecto, la OMS/OPS expone algunas razones que se definen como 'causales de la fragmentación' actual, entre las que se encuentran la segmentación del sistema de salud y la descentralización de los servicios de salud en todos los niveles de atención [8], el enfoque de atención basado en planes de beneficio y los niveles de responsabilidad entre sector público y privado, todo lo cual coadyuva a la materialización de los EA.

\section{Conclusión}

Las experiencias presentadas permiten observar que la identificación de los riesgos en SP en APS habitualmente se realiza de la misma manera que se hace en la gestión clínica hospitalaria, sin tener en cuenta que los riesgos son diferentes, aunque en Colombia no existe una directriz normativa legar para hacerlo. Probablemente, esto ocasione un sesgo que no permita una adecuada identificación de riesgos, ya que las percepciones de los profesionales de salud que reportan pueden variar de acuerdo con el escenario donde se efectúen. Tal vez esta sea la razón por la cual la SP y la gestión de riesgos en APS podrían quedarse en una actividad en el olvido. Aunque es posible que existan algunas iniciativas aisladas por parte de algunos actores del SGSSS para la identificación de riesgos de SP en el ámbito de la APS, su enfoque continúa siendo prematuro, teniendo en cuenta que, en primer lugar, existen vacíos en la política pública y en el marco normativo que la materializa y, en segundo lugar, no se cuenta con sistemas de notificación en tiempo real que consoliden de manera pertinente los EA o incidentes que se presentan en el ámbito de la APS. En tercer lugar, existe una mayor limitación en cuanto a una estandarización de términos (taxonomía) que permita clasificar adecuadamente los incidentes o EA en APS, lo que hace que los resultados sean muy variados en la determinación de su frecuencia [37]. En cuarto lugar, la educación y la sensibilización de los profesionales de la salud en conceptos propios de la SP es limitada. Y, en quinto lugar, el temor a las represalias del personal de salud ante la notificación de los EA por parte de las instituciones, lo cual podría repercutir gravemente en la cultura de la SP. Por lo anterior, es necesario el compromiso de la alta gerencia de las organizaciones de salud, a efectos de construir los espacios necesarios para transitar de una cultura punitiva a una cultura proactiva y del aprendizaje organizacional, a partir de la aceptación de los errores. En cuanto al sexto y último aspecto, hay vacíos en la inclusión de los pacientes y sus familias en la identificación y prevención de EA, porque de acuerdo con la estructura organizativa de la APS, la mayor participación en el cuidado de la salud la debe asumir el paciente y su familia. Todo ello significa que la SP no solamente debe ser enfocada en los servicios asistenciales 
o en la atención del paciente agudo, ya que las dimensiones por evaluar están relacionadas directamente con el entorno hospitalario, como la seguridad de los equipos, la prevención de la infecciones asociadas al cuidado de la salud, la seguridad en el uso de medicamentos desde la prescripción, dispensación y administración, procedimientos y prácticas clínicas, y la comunicación entre los profesionales de la salud, entre otras. Esto significa que, aun cuando la incidencia de EA en APS sea de menor proporción que la ocurrida en el ámbito hospitalario, y a pesar de que en Colombia no existan cifras oficiales al respecto, esto no implica que los eventos que se presenten no sean potencialmente graves, incluso letales, para los pacientes. De esta manera, pareciera que la seguridad del paciente y la identificación de riesgos en APS no contara con el suficiente peso político y epidemiológico para tenerlos en cuenta y que todas las miradas de los actores del SGSSS priorizaran la gestión de riesgos en los servicios de media y alta complejidad. Por ello, conviene considerar el reto de integrar la evaluación de los riesgos que los pacientes enfrentan durante el proceso de APS, que es la puerta de entrada a los servicios de salud. Para cumplir este propósito, es necesario que el Estado formule la política pública pertinente y que las instituciones implementen métodos estandarizados para cuantificar y clasificar los riesgos en APS, de conformidad con los contextos específicos donde se presenten, y que estén diseñados para este fin. El SGSSS no debe ser ajeno a estos cambios, especialmente cuando se prevén grandes transformaciones en el modelo de salud y aseguramiento con la reglamentación de la Ley 1751 de 2015 - Ley Estatutaria de Salud, "por la cual se regula el derecho fundamental a la salud," con una apuesta por la APS y un mejor enfoque en la promoción salud y prevención de la enfermedad. Esto permitirá tener un goce efectivo de la salud al romper con un sistema en el que las patologías tienen diagnósticos tardíos, con atención de mayor costo y una mayor mortalidad [38].

Por consiguiente, se tiene que entender que los cambios no sucederán de un momento a otro, son lentos, pero el objetivo es que sean hacia un bien dirigido [36]. Por esta razón, la PAIS es el resultado de una política de reordenamiento de la Ley 100 para "recuperar la confianza pública en el sistema de salud", deteriorada por la profunda crisis en que se encuentra, y para redefinir "el rol del asegurador", principalmente a través de a) el fortalecimiento de la gestión del riesgo financiero; b) asignarle a las aseguradoras la implementación de la APS y la coordinación de la prestación de servicios en todos los niveles a través de redes integradas; y c) estableciendo que "El asegurador debe actuar en cada territorio en lo referente a la planeación, ejecución y seguimiento de los planes territoriales de salud" [9].

En pocas palabras, el Ministerio de Salud y Protección Social, en el marco estratégico de la PAIS, indica que la AP debe tener un mayor alcance que el componente asistencial de los servicios curativos, tal como lo plantea la OMS en su informe 2008, está orientada a fomentar la salud y la calidad de vida de la población, y no solo a la recuperación de la enfermedad [5]. Si es así, ¿ cuáles serían los grandes retos que tendría que asumir el Ministerio de Salud y Protección Social (MSPS) para garantizar accesibilidad a los servicios de APS, siendo la entrada al sistema de salud, para garantizar entornos seguros y evitar la ocurrencia de eventos adversos? Si se tiene en cuenta que el SGSSS está migrando hacia un modelo de salud enfocado en la prevención, donde el individuo deberá tomar conciencia de la importancia de adquirir hábitos saludables y propender por reducir los riesgos en su salud, ¿cuáles serían las herramientas más adecuadas para evaluar los riesgos en seguridad del paciente en el ámbito de la APS con las nuevas políticas y estrategias implementadas por el MSPS?

Son muchos los interrogantes y grandes retos los que nos esperan, bien vale la pena reflexionar sobre ellos.

\section{Referencias}

1. Ayuso-Murillo D, de Andrés-Gimeno B, Noriega-Matanza C, López-Suárez RJ, Herrera-Peco I. Gestión de la calidad, un enfoque directivo para la seguridad del paciente. Enfermería Clínica. 2017; 27(4): 251-5.

2. Casanova YG, Díaz SN, Pérez AM. La seguridad del paciente como paradigma de la excelencia del cuidado en los servicios de salud. Revista Cubana de Enfermería. 2014; 30(1):62-4. 
3. Torijano-Casalengua ML, Astier-Peña P, Mira-Solves JJ. El impacto que tienen los eventos adversos sobre los profesionales sanitarios de atención primaria y sus instituciones. Atención Primaria. 2016; 48(3):143-6.

4. Cruz-Peñate M. La Atención Primaria de Salud, contexto histórico, conceptos, enfoques y evidencias. Bogotá, D. C.: Ministerio de la Protección Social, 2011.

5. Colombia, Ministerio de Salud y Protección Social. Política de Atención Integral en Salud Ministerio de Salud y Protección Social. Bogotá: Ministerio de Salud y Protección Social; 2016.

6. Macinko J, Montenegro H, Nebot Adell C, Etienne CF. La renovación de la atención primaria de salud en las Américas. Washington: OPS: 2007.

7. Brommet A, Lee J, Serna JA. Atención primaria: Una estrategia renovada. Colombia Médica 2011;42(3):379-87.

8. Franco-Giraldo Á. Referentes teóricos para el análisis de la reforma del sistema de salud colombiano. Revista Gerencia y Políticas de Salud. 2012; 11(22):28-42.

9. Useche-Aldana B. La salud en el Plan Departamental de Desarrollo “Santander Nos Une”. MedUNAB, 19(1) 2016; 19(1):33-9.

10. Ubeda SG. ¿Se necesita un esfuerzo para reemplazar la cultura punitiva por la de seguridad del paciente? Revista de Calidad Asistencial. 2016;31(3):173-6.

11. Rojas-Armadillo ML, Jiménez-Báez MV, Chávez-Hernández MM, González-Fondón A. Validación de un sistema de registro de eventos adversos en atención primaria. Revista Médica del Instituto Mexicano del Seguro Social 2016;54(3):327-33.

12. Rodríguez-Cogollo R, Paredes-Alvarado I, Galicia-Flores T, Barrasa-Villar J, Castán-Ruiz S. Cultura de seguridad del paciente en residentes de medicina familiar y comunitaria de Aragón. Revista de Calidad Asistencial. 2014; 29(3):143-9.

13. Castro JAP, Vázquez CMC, Vázquez CM, Domínguez-De La Peña MA, Jiménez-Díaz IB, Rueda-Rodríguez A. Los pequeños grandes detalles de la seguridad del paciente. Revista Conamed. 2012; 17(3):130-5.

14. Luengas S. Modelo para gestionar la seguridad de los pacientes en las instituciones de Salud. Vía Salud. 2008; (43).

15. Milos P, Larraín AI. La vinculación ético-jurídica entre la gestión del cuidado y la gestión de riesgos en el contexto de la seguridad del paciente. Aquichan. 2105; 15(1):141-53.

16. Pais Iglesias B, Rodríguez Pérez B, Rodríguez M, Carreras Viñas M. Gestión de segundas víctimas en Galicia. Revista de Calidad Asistencial. 2016; 31(Supl. 2):47-9.

17. Posada Saldarriaga MF, Sánchez García ÓE. Comprensiones de un modelo para la educación en ambientes virtuales en las prácticas de enseñanza de docentes: Diplomatura en Docencia Universitaria con énfasis en Ambientes Virtuales de Enseñanza y de Aprendizaje de la Universidad Pontificia Bolivariana. Revista Q 2015; 9(18).

18. Buenas prácticas para la seguridad del paciente en la atención en salud. Mejorar la seguridad en los procedimientos quirúrgicos [internet] [acceso: 23 de diciembre de 2016]. Disponible en: http://www.scsalud.es/documents/2 162705/2163013/Manual+de+Seguridad+del+Paciente+Quir\%C3\%BArgico+v4.pdf.

19. Agra-Varela Y, Fernández-Maíllo M, Rivera-Ariza S, Sáiz-Martínez-Acitorez I, Casal-Gómez J, Palanca-Sánchez I, et al. Red Europea de Seguridad del Paciente y Calidad. Desarrollo y resultados preliminares en Europa y en el Sistema Nacional de Salud. Revista de Calidad Asistencial. 2015; 30(2):95-102.

20. Santacruz-Varela J, Rodríguez-Suárez J, Fajardo-Dolci G, Hernández-Torres F. Historia de los sistemas de notificación y registro de incidentes. Revista Conamed. 2012;17(2):77-80.

21. Organización Mundial de la Salud (OMS). Seguridad del paciente. Métodos e instrumentos de medición en la investigación sobre seguridad del paciente [internet]. 2016. Disponible en: http://www.who.int/patientsafety/ research/methods_measures/es/

22. Martin LD, Rampersad SE, Low DKW, Reed MA. Mejoramiento de los procesos en el quirófano mediante la aplicación de la metodología Lean de Toyota. Revista Colombiana de Anestesiología, 2014; 42(3):220-8.

23. Panesar SS, Carson-Stevens A, Cresswell KM, Salvilla SA, Slight SP, Javad S, et al. How safe is primary care? A systematic review. BMJ Qual Saf. 2015. 
24. Muñoz FP, Marín VP. Evaluación de la cultura de seguridad del paciente en el ámbito de un área sanitaria. Revista de Calidad Asistencial. 2013; 28(6):329-36.

25. Zapata Álvarez F, Ospina Moreno J, Sepúlveda Castaño M, López Murillo E. Prototipo de evaluación con TIC: un paso hacia el cambio curricular. Revista Trilogía. 2013; (8):93-106.

26. Valdés Cuervo AA, Sánchez PA. Las creencias de los docentes acerca de la participación familiar en la educación. Revista Electrónica de Investigación Educativa. 2016;18(2):105-15.

27. Silveira H. La participación de las familias en los centros educativos. Un derecho en construcción. Revista Electrónica Interuniversitaria de Formación del Profesorado 2016; 19(1):17-29.

28. Delgado MM, de Cos PM, Rodríguez GS, Rodríguez JÁ, Cía IG, Azuara BO, et al. Análisis de los factores contribuyentes en incidentes relacionados con la seguridad del paciente en Medicina Intensiva. Medicina Intensiva. 2015; 39(5):263-71.

29. Torijano-Casalengua ML, Olivera-Cañadas G, Astier-Peña MP, Maderuelo-Fernández JÁ, Silvestre-Busto C. Validación de un cuestionario para evaluar la cultura de seguridad del paciente de los profesionales de atención primaria en España. Atención Primaria. 2013; 45(1):21-37.

30. Merino-Plaza MJ, Carrera-Hueso FJ, Roca-Castelló MR, Morro-Martín MD, Martínez-Asensi A, Fikri-Benbrahim N. Relación entre la satisfacción laboral y la cultura de seguridad del paciente. Gaceta Sanitaria. 2017.

31. Beltrán-Salazar OA. Las instituciones de salud no favorecen el cuidado Significado del cuidado humanizado para las personas que participan directamente en él. Investigacion y Educacion en Enfermería. 2014; 32(2):194-205.

32. Colombia, Ministerio de Salud y Protección Social. Política de Seguridad del Paciente. Disponible en: https://ww w.minsalud.gov.co/salud/PServicios/Paginas/Seguridad-del-Paciente.aspx

33. Catalán A, Borrell F, Pons À, Amado E, Baena JM, Morales V. Seguridad del paciente en atención primaria: proyecto PREFASEG (PREscripción FArmacológica SEGura). Medicina Clínica. 2014; (143):32-5.

34. Torres-Ricarte M, Crusat-Abelló E, Peñuelas-Rodríguez S, Zabaleta-del-Olmo E. El Nurse-led en la Atención Primaria de Salud: una oportuna y prometedora innovación organizativa. Enfermería Clínica. 2015; 25(2):87-91.

35. Dorado AC, Cubero CG, Ferradal IG, Safont TA, Márquez MS, Requejo SS, et al. Identificación de las prácticas seguras simples en un área de atención primaria. Revista de Calidad Asistencial. 2011; 26(5):292-8.

36. Cañadas GO, Dorado AC, Canela MD, Fernández-Martínez B, León GO, Ballesteros MC. Identificación de eventos centinela en atención primaria. Revista de Calidad Asistencial. 2017; 32(5): 269-77.

37. Ortiz Salazar PA, Quintero Higuera EF, Valdés Puentes CE. ¿Por qué se debe renovar la atención primaria en salud? Revista CES Salud Pública. 2013; 4(1):77-81.

38. Perales Mejía FJ, Escobedo Carrillo MM. La participación social en la educación: entre propuestas innovadoras y tradición educativa. Revista Electrónica de Investigación Educativa. 2016;18(1):69-81.

\section{Notas}

* Artículo de reflexión.

\section{Licencia Creative Commons CC BY 4.0}

Como citar este artículo: Moya Sáenz OL. La seguridad del paciente en atención primaria en salud. ¿Una actividad que podría quedar en el olvido? Rev Gerenc Polít Salud. 2017; 17(34): 1-14. https:// doi.org/10.11144/Javeriana.rgps17-34.apap 